\title{
On a Resource Based Ecological Competition Model with Interference
}

\author{
Sze-Bi Hsu* \\ Department of Mathematics, University of Utah, Salt Lake City, UT 84112, USA
}

\begin{abstract}
A resource based ecological competition model with interference is proposed. The model is based on Lotka-Volterra dynamics with two predators competing for a single, limited prey. Interference effects are considered in this article. When the interference coefficient, expressing the damage effect from its rival, is small, the mathematical analysis shows that the winner in purely exploitative competition still outcompetes its rival. However, if the interference coefficient is large enough then the competition outcome will depend on initial population of predator species.
\end{abstract}

Key words: Predator-prey system - Competition - Interference

\section{Introduction}

The classical theory of ecological competition between two or more species, attributed to Lotka and Volterra [11], is an extension of the basic logistic model of single-species growth that dates from Verhulst [10]. The dynamical equations for this theory for two competitors, 1 and 2, are often written as:

$$
\begin{aligned}
& d N_{1} / d t=r_{1} N_{1}\left(1-\left(N_{1}+\alpha N_{2}\right) / K_{1}\right) \\
& d N_{2} / d t=r_{2} N_{2}\left(1-\left(\beta N_{1}+N_{2}\right) / K_{2}\right),
\end{aligned}
$$

where $N_{i}$ is the number of the $i$ th competing species, $r_{i}$ and $K_{i}$ are the intrinsic rate of increase and the carrying capacity of the $i$ th competitor, respectively, and $\alpha$ and $\beta$ are the interaction or "competition" coefficients, expressing the per capita competitive effect of species 2 on 1 , and 1 on 2 , on the growth rate and realized carrying capacity of the rival species. In the absence of competition $(\alpha=\beta=0)$, each population grows to its respective carrying capacity. In the presence of competition, one or the other rival may survive while its competitor dies out, or else the rivals may coexist. In the two-species case, there are four possible outcomes

* Present address: Department of Applied Mathematics, National Chiao-Tung University, Hsin-Chu, Taiwan 
provided that the initial populations are both positive; which outcome occurs depends on the carrying capacities and competition coefficients. Competitive stability (coexistence) occurs when $\alpha<K_{1} / K_{2}$ and $\beta<K_{2} / K_{1}$, competitive instability (initial number of each rival determines winner) occurs when these inequalities are both reversed, and competitive dominance (one or the other species wins regardless of initial numbers) occurs when one but not both of these inequalities are reversed. A pervasive problem with classical theory is that it is "phenomenological", seeking to describe how the numbers of competitors change without ever being specific about which resources are the focus of competition, nor about how efficiently the rivals exploit or control these limited resources.

During the last 20 years increasing attention has been given to the details of the processes underlying consumer-resource interactions, with the goal of constructing more mechanistic theories of interspecific competition. Research has been focused on three principal questions. First, do the rival species compete only indirectly by lowering the shared pool of limited resources (exploitative competition), or do they also compete more directly by harming their rivals or by sequestering some of the resources for their exclusive use (interference competition) [8]? Second, how efficiently do the rivals exploit these limiting resources? In particular, how do the per capita consumption rates of each species respond to a change in resource concentration (nutrients, prey, etc.) in the environment ("functional" response)? Finally, how do these resources, once consumed, translate into a particular rate of population growth ("numerical" response)?

In this article we present a mathematical model with two species exploiting a single, limited resource and interfering each other directly at the same time. In theoretical ecology the distinction between exploitation and interference is illdefined and not absolute. Sometimes a species can be exploiting and interfering at the same time [6]. Especially interference competition, while common in nature, is mediated through a diversity of mechanisms. As yet there is little consensus about the way that interference should be modeled mathematically given that the effects of toxins or injury are so varied, and are different from the effects of resource sequestering. In order to understand this complicated problem, we choose to model the simplest possible predator (consumer) functional response to increasing prey (resource) density. This is the response in which the feeding rate of the predator increases linearly with prey density (Type I under Holling's [4] classification). The interference effect of the predator species on the growth of its rival is assumed to be proportional to its current population.

The remainder of this paper is organized as follows. A statement of the model and definition of the parameters appears in section 2. The analytical results are given without proof in section 3 . The proofs are deferred to section 4 . Section 5 is the discussion section.

\section{Statement of the Model}

The present analysis concerns the behavior of a predator-prey system consisting of two predator species, $N_{1}$ and $N_{2}$ and one prey species $R$. We specifically assume both predator species have access to prey and compete not only by lowering the population of shared prey but also by interfering with its rival. The interference 
effect of the predator species on the growth of its rival is assumed to be proportional to its number present. Death rates are assumed to follow a "Type III" survivorship [9] in which the number dying is proportional to the number currently alive. We also assume that there are no significant time lags in the system, that growth rate is logistic in the prey species in the absence of predation, and that the functional response of the predator is linear. With these assumptions the model is given by:

$$
\begin{aligned}
R^{\prime} & =R\left[r\left(1-\frac{R}{K}\right)-k_{1} N_{1}-k_{2} N_{2}\right] \\
N_{1}^{\prime} & =N_{1}\left[b_{1} R-D_{1}-\alpha_{21} N_{2}\right] \\
N_{2}^{\prime} & =N_{2}\left[b_{2} R-D_{2}-\alpha_{12} N_{1}\right] \\
R(0) & >0, \quad N_{i}(0)>0 \text { for } i=1,2
\end{aligned}
$$

where

$$
'=\frac{d}{d t}, \quad t=\text { time, }
$$

$R(t)=$ the number of prey species at time $t$, $N_{i}(t)=$ the number of $i$ th predator at time $t$,

$r=$ the intrinsic rate of increase for prey species,

$K=$ the carrying capacity for the prey species,

$k_{i}=$ the feeding rate per predator (predator species $i$ ) per unit consumed,

$b_{i}=$ the birth rate per predator (predator species $i$ ) per unit prey consumed,

$D_{i}=$ the death rate of $i$ th predator

$\alpha_{i j}=$ the interference coefficient measuring the damage effect of predator species $i$ on predator species $j$.

It is noted that the coefficients $K, k_{i}, b_{i}, D_{i}$ are strictly positive and $\alpha_{12}, \alpha_{21}$ are nonnegative.

We analyze the behavior of solution of this system of ordinary differential equations, in order to answer the biological question: Under what conditions will neither, one or both species of predator survive or die out? If only one predator survives, we also seek to determine the limiting behavior of the surviving predator and its prey.

\section{Statement of Results}

In this section we state the principal results of the paper. The proofs are deferred to the next section. The first lemma is a statement that the system given by (2.1) is as "well-behaved" as one infers from the biological problem. It is easy to prove the lemma and we omit it.

Lemma 3.1. Solutions of (2.1) are positive and bounded. Furthermore, for any $\varepsilon>0$ there exists $t_{0}>0$ such that $R(t) \leqslant K+\varepsilon$ for $t \geqslant t_{0}$.

The next lemma provides conditions under which the predators cannot survive on the prey, given the carrying capacity of each prey population, even in the absence of competition. The proof for this lemma follows directly from (2.1) and Lemma 3.1 and we omit it. 
Lemma 3.2. A necessary condition for either predator species $N_{i}$ to survive is $b_{i} K>D_{i}$.

This lemma states if the death rate $D_{i}$ of the $i$ th predator is too high or if the carrying capacity $K$ of the prey species is too small to support the $i$ th predator, then the $i$ th predator will die out. We note the result is independent of competition and interference.

We state the principal result in the case of inadequate carrying capacity of prey in three parts. We are able in Theorem 3.3 to determine the asymptotic behavior of the solutions. The theorem may be summarized as the unsuccessful competitor does not affect the eventual behavior of the survivor and its prey.

Theorem 3.3. Let $\lambda_{i}=D_{i} / b_{i}, i=1,2$.

(i) If $\lambda_{i}>K$ for $i=1,2$, then $\lim _{t \rightarrow \infty} R(t)=K, \lim _{t \rightarrow \infty} N_{i}(t)=0, i=1,2$.

(ii) If $\lambda_{1}<K<\lambda_{2}$ then the trajectory of (2.1) approaches the equilibrium $\left(E_{1}\right)$ as $t \rightarrow \infty$ where

$$
\left(E_{1}\right)=\left(\lambda_{1}, N_{1}^{*}, 0\right), \quad N_{1}^{*}=\frac{r}{k_{1}}\left(1-\frac{\lambda_{1}}{K}\right) .
$$

(iii) If $\lambda_{2}<K<\lambda_{1}$ then the trajectory of (2.1) approaches the equilibrium $\left(E_{2}\right)$ as $t \rightarrow \infty$ where

$$
\left(E_{2}\right)=\left(\lambda_{2}, 0, N_{2}^{*}\right), \quad N_{2}^{*}=\frac{r}{k_{2}}\left(1-\frac{\lambda_{2}}{K}\right) .
$$

In order to discuss the interior equilibrium point, we may assume as a basic hypothesis:

$$
\lambda_{1}<K, \quad \lambda_{2}<K .
$$

Under this assumption, the equations of (2.1) may be relabeled without loss of generality, so that we assume

$$
\lambda_{1}<\lambda_{2}<K .
$$

The following theorem is our main results concerning the effect of interference.

Theorem 3.4. Let $(\mathrm{H})$ hold and $\alpha_{21}>0, \alpha_{12} \geqslant 0$.

(i) If $r\left(1-\lambda_{2} / K\right)<\left(k_{2} / \alpha_{21}\right)\left(b_{1} \lambda_{2}-D_{2}\right)$ then the equilibrium $\left(E_{1}\right)$ is globally asymptotically stable in the interior of the first octant.

(ii) If $r\left(1-\lambda_{2} / K\right)>\left(k_{2} / \alpha_{21}\right)\left(b_{1} \lambda_{2}-D_{2}\right)$ then the unique interior equilibrium $\left(E_{c}\right)=\left(R_{c}, N_{1 c}, N_{2 c}\right)$ exists and is unstable where

$$
\begin{gathered}
K>R_{c}=\frac{r \alpha_{21} \alpha_{12}+k_{2} \alpha_{12} D_{1}+k_{1} \alpha_{21} D_{2}}{\frac{r}{K} \alpha_{21} \alpha_{12}+k_{2} b_{1} \alpha_{12}+k_{1} b_{2} \alpha_{21}} \geqslant \lambda_{2}, \\
N_{2 c}=\frac{1}{\alpha_{21}}\left(b_{1} R_{c}-D_{1}\right)>0, \\
N_{1 c}=\frac{1}{k_{1}}\left(r\left(1-\frac{R_{c}}{K}\right)-k_{2} N_{2 c}\right)>0 .
\end{gathered}
$$


Furthermore, in this case the equilibria $\left(E_{1}\right)$ and $\left(E_{2}\right)$ are both asymptotically stable and there exists a two-dimensional stable manifold through $\left(E_{c}\right)$.

\section{Proof}

Before we explore the behavior of the solutions of (2.1), we need the following lemmas. The proofs of (i) and (ii) of Lemma 4.2 are straightforward and are omitted. The proof of (iii) of Lemma 4.2 comes from [5].

Lemma 4.1 (Coppel [2, p. 141]). If a function $f(t)$ has a finite limit as $t \rightarrow \infty$ and $f^{(n)}(t)$ (the nth derivative) is bounded for $t \geqslant t_{0}$, then $\lim _{t \rightarrow \infty} f^{(k)}(t)=0,0<k<n$.

Lemma 4.2. (i) If $\lim _{t \rightarrow \infty} N_{i}(t)=0, i=1,2$, then $\lambda_{i} \geqslant K, i=1,2$.

(ii) Let $(\mathrm{H})$ hold. Then the equilibrium $\left(E_{1}\right)$ is asymptotically stable.

(iii) The solution of $(2.1)$ with $R(0)>0, N_{1}(0)>0, N_{2}(0)=0$ satisfies $\lim _{t \rightarrow \infty} R(t)=\lambda_{1}, \lim _{t \rightarrow \infty} N_{1}(t)=N_{1}^{*}$ and $N_{2}(t) \equiv 0$.

Proof of Theorem 3.3. Part (i) follows directly from Lemma 3.2 and the first equation of (2.1). We only need to prove (ii). The proof of (iii) is similar to that of (ii).

From Lemma 3.2, Lemma 4.2 (i) and (H), it follows that $\lim _{t \rightarrow \infty} N_{2}(t)=0$ and $\lim \sup _{t \rightarrow \infty} N_{1}(t)>0$. If $\lim _{t \rightarrow \infty} N_{1}(t)$ exists and is equal to $c>0$, then since by Lemma 3.1, $N_{1}^{\prime \prime}(t)$ is bounded, Lemma 4.1 implies $\lim _{t \rightarrow \infty} R(t)=\lambda_{1}$. Again using Lemma 3.1, $R^{\prime \prime}(t)$ is bounded and hence $\lim _{t \rightarrow \infty} R^{\prime}(t)=0$ and $c=N_{1}^{*}$.

If $\lim _{t \rightarrow \infty} N_{1}(t)$ does not exist, choose a sequence $\left\{t_{n}\right\}$ such that $\lim _{t \rightarrow \infty} t_{n}=\infty, N_{1}\left(t_{n}\right)$ is a relative maximum, $N_{1}\left(t_{n}\right)>\varepsilon$ for some $\varepsilon>0$, for all $n$ and $\lim _{n \rightarrow \infty} N_{1}\left(t_{n}\right)=N_{1 \omega}$ for some $N_{1 \omega} \geqslant \varepsilon>0$. By (2.1), we have $\lim _{n \rightarrow \infty} R\left(t_{n}\right)=\lambda_{1}$. Then $\left(\lambda_{1}, N_{1 \omega}, 0\right) \in \Omega$ where $\Omega$ is the omega limit set of the solution ( $\left.R(t), N_{1}(t), N_{2}(t)\right)$ of (2.1) and lies on the $R-N_{1}$ plane. Using Lemma 4.2 (iii), it follows that the solution of (2.1) with $R(0)=\lambda_{1}, N_{1}(0)=N_{1 \omega}, N_{2}(0)=0$ satisfying $\lim _{t \rightarrow \infty} R(t)=\lambda_{1}, \lim _{t \rightarrow \infty} N_{1}(t)=N_{1}^{*}$ and $N_{2}(t) \equiv 0$. This and the invariance property of the omega limit set [3] imply that $\left(\lambda_{1}, N_{1}^{*}, 0\right) \in \Omega$. However, $\left(\lambda_{1}, N_{1}^{*}, 0\right)$ is asymptotically stable by Lemma 4.2 (ii). Hence the trajectory $\left(R(t), N_{1}(t), N_{2}(t)\right)$ approaches the equilibrium $\left(E_{1}\right)=\left(\lambda_{1}, N_{1}^{*}, 0\right)$. In particular, $\lim _{t \rightarrow \infty} N_{1}(t)=N_{1}^{*}$. This is the desired contradiction.

Proof of Theorem 3.4 (i). First we show that $\lim _{t \rightarrow \infty} R(t) N_{2}(t)=0$. Let $\xi>0, \eta>0$ be selected below.

$$
\begin{aligned}
\frac{R^{\prime}(t)}{R(t)}+ & \xi \frac{N_{2}^{\prime}(t)}{N_{2}(t)}-\eta \frac{N_{1}^{\prime}(t)}{N_{1}(t)} \\
= & r\left(1-\frac{R(t)}{K}\right)-k_{1} N_{1}(t)-k_{2} N_{2}(t)+\xi\left(b_{2} R(t)-D_{2}-\alpha_{12} N_{1}(t)\right) \\
& -\eta\left(b_{1} R(t)-D_{1}-\alpha_{21} N_{2}(t)\right) \\
\leqslant & r\left(1-\frac{R(t)}{K}\right)-k_{2} N_{2}(t)+\xi\left(b_{2} R(t)-D_{2}\right)-\eta\left(b_{1} R(t)-D_{1}-\alpha_{21} N_{2}(t)\right) \\
= & \left(r-\xi D_{2}+\eta D_{1}\right)+R(t)\left(-\frac{r}{K}+\xi b_{2}-\eta b_{1}\right)+N_{2}(t)\left(\eta \alpha_{21}-k_{2}\right) .
\end{aligned}
$$


Let $\eta=k_{2} / \alpha_{21}$ and choose $\xi>0$ satisfying $r-\xi D_{2}+\eta D_{1}<0$ and $-r / K+\xi b_{2}-\eta b_{1}<0$, i.e.,

$$
\xi>\frac{1}{D_{2}}\left(r+\frac{k_{2} D_{1}}{\alpha_{21}}\right) \quad \text { and } \quad \xi<\frac{1}{b_{2}}\left(\frac{r}{k}+\frac{k_{2}}{\alpha_{21}} b_{1}\right) .
$$

Since

$$
r\left(1-\frac{\lambda_{2}}{K}\right)<\frac{k_{2}}{\alpha_{21}}\left(b_{1} \lambda_{2}-D_{1}\right)
$$

is equivalent to

$$
\frac{1}{b_{2}}\left(\frac{r}{K}+\frac{k_{2}}{\alpha_{21}} b_{1}\right)>\frac{1}{D_{2}}\left(r+\frac{k_{2} D_{1}}{\alpha_{21}}\right),
$$

we can choose $\xi$ satisfying the above inequalities. Let $\xi^{*}=r-\xi D_{2}+D_{1}<0$. Integrating from 0 to $t$ and taking exponentials on both sides of (4.1) yields

$$
\left(\frac{R(t)}{R_{0}}\right) \cdot\left(\frac{N_{2}(t)}{N_{2}(0)}\right)^{\xi} \leqslant\left(\frac{N_{1}(t)}{N_{1}(0)}\right)^{\eta} \cdot e^{\xi * t} \leqslant M e^{\xi^{*} t}
$$

It follows that $\lim _{t \rightarrow \infty} R(t)\left(N_{2}(t)\right)^{\check{\zeta}}=0$. We claim $\lim _{t \rightarrow \infty} R(t) N_{2}(t)=0$. If $\xi>1$, then $\left(R(t) N_{2}(t)\right)^{\xi}=(R(t))^{\xi-1} \cdot R(t)\left(N_{2}(t)\right)^{\xi} \leqslant\left(\sup _{0 \leqslant t \leqslant \infty} R(t)\right)^{\xi-1} \cdot R(t) N_{2}(t)^{\xi} \rightarrow 0$ as $t \rightarrow \infty$. If $\xi<1$, then $R(t) N_{2}(t)=\left(N_{2}(t)\right)^{1-\xi} . R(t)\left(N_{2}(t)\right)^{\xi} \leqslant\left(\sup _{0 \leqslant t<\infty} N_{2}(t)\right)^{1-\xi}$. $R(t)\left(N_{2}(t)\right)^{\xi} \rightarrow 0$ as $t \rightarrow \infty$. Hence $\lim _{t \rightarrow \infty} R(t) N_{2}(t)=0$.

Next we show that $\lim _{t \rightarrow \infty} N_{2}(t)=0$. From (2.1) it follows that $N_{2}^{\prime}(t) \leqslant b_{2} N_{2}(t) R(t)-D_{2} N_{2}(t)$. Then $\lim _{t \rightarrow \infty} N_{2}(t) R(t)=0$ yields $\lim _{t \rightarrow \infty} N_{2}(t)$ $=0$. Using the arguments in Theorem 3.3 yields the conclusion of (i).

Proof of Theorem 3.4 (ii). Under the assumption

$$
r\left(1-\frac{\lambda_{2}}{K}\right)>\frac{k_{2}}{\alpha_{21}}\left(b_{1} \lambda_{2}-D_{1}\right)
$$

and $(\mathrm{H})$, we have $K>R_{c} \geqslant \lambda_{2}$. Hence the existence and uniqueness of the equilibrium $\left(E_{c}\right)$ follows directly from algebraic computation. From an elementary linear stability analysis about $\left(E_{c}\right)$, the characteristic polynomial for the variational matrix evaluated at $\left(E_{c}\right)$ is

$$
f(z)=z^{3}+A_{1} z^{2}+A_{2} z+A_{3}
$$

where

$$
\begin{aligned}
& A_{1}=\frac{r}{K} R_{c}>0, \\
& A_{2}=k_{2} b_{2} X_{2 c} R_{c}+k_{1} b_{1} X_{1 c} R_{c}-\alpha_{21} \alpha_{12} X_{1 c} X_{2 c}, \\
& A_{3}=-\frac{r}{K} \alpha_{12} \alpha_{21}-k_{1} b_{2} \alpha_{21}-k_{2} b_{1} \alpha_{12}<0 .
\end{aligned}
$$

Since $A_{1}>0$ and $A_{3}<0$. From the Routh-Hurwitz criterion, $\left(E_{c}\right)$ is unstable. 
Among three roots of $f(z)=0$, one is positive, real and the other two, real or complex, have negative real parts. Hence $\left(E_{c}\right)$ is an unstable saddle point and there exists a two-dimensional stable manifold through $\left(E_{c}\right)$ [3].

From Lemma 4.2 (ii), the equilibrium $\left(E_{1}\right)$ is always asymptotically stable. The asymptotic stability of $\left(E_{2}\right)$ simply follows from the assumption

$$
r\left(1-\frac{\lambda_{2}}{K}\right)>\frac{k_{2}}{\alpha_{21}}\left(b_{1} \lambda_{2}-D_{2}\right)
$$

and linear stability analysis about $\left(E_{2}\right)$.

\section{Discussion}

Exploitative competition and interference competition are common in nature. Exploitation is usually defined as the ability of a species to find, occupy and retain vacant vital resources. Interference is regarded in its pure form as the ability of a species to damage another either directly by attacking its individuals or indirectly by harming its resources or blocking its access to them. However, the distinction between these two forms of interspecies competition is not absolute. Sometimes a species can be exploiting and interfering at the same time. Brian [1] refers to interference and exploitation as "isolated components of the dual competition concept" and concludes that the Lotka-Volterra model (1.1) involves competition by interference alone while the Windsor model [12] (i.e. the second and third equations in (2.1) with $\alpha_{12}=\alpha_{21}=0$ ) involves only exploitation.

In this paper we combine these two models into our model (2.1). We assume two predator species are competing for a single prey species which grows logistically and the predators consume prey according to a linear functional response. The massaction formulation of feeding rate is most accurate at low prey densities. In addition, we assume these two predator species interfere with each other directly. The interference effect of a predator species on the growth of its reval is assumed to be proportional to its current population. Under the assumption $(H)$, if the competition is purely exploitative, i.e., $\alpha_{12}=\alpha_{21}=0$, predator species $N_{1}$ is always the winner [5] (for the case $\alpha_{12}=\alpha_{21}=0$, A. Leung [7] discusses the same problem including even $\lambda_{1}=\lambda_{2}$ ). Taking into account the interference effects, Theorem 3.4 says if the interference coefficient $\alpha_{21}$ is small, i.e., $r\left(1-\lambda_{2} / K\right)<$ $\left(k_{2} / \alpha_{21}\right)\left(b_{1} \lambda_{2}-D\right)$, species $N_{1}$ still outcompetes species $N_{2}$ even if species $N_{1}$ does not interfere with species $N_{2}$ (i.e., $\alpha_{12}=0$ ). However if $\alpha_{21}$ is sufficiently large, i.e., $r\left(1-\lambda_{2} / K\right)>\left(k_{2} / \alpha_{21}\right)\left(b_{1} \lambda_{2}-D_{1}\right)$, then the initial populations of the rival predator species influence the outcome of competition. We note that large $\alpha_{21}$ does not guarantee that species $N_{2}$ will outcompete species $N_{1}$ provided the initial population of $N_{2}$ is smaller compared to that of $N_{1}$.

\section{References}

1. Brian, M. V.: Exploitation and interference in interspecies competition. J. Anim. Ecol. 25, 339-347 (1956)

2. Coppel, W. A.: Stability and asymptotic behavior of differential equations. Boston: Heath 1965.

3. Hale, J. K.: Ordinary differential equations. New York: Wiley-Interscience 1969 
4. Holling, C. S.: The functional response of predators to prey density and its role in mimicry and population regulation. Mem. Ent. Soc. Can. 45, 1-60 (1965)

5. Hsu, S. B.: Limiting behavior of competing species. SIAM J. Applied Math. 34, 760-763 (1978)

6. Hutchinson, G. E.: An introduction to population ecology, p. 140. Yale University Press, 1978

7. Leung, A.: Limiting behavior for several interacting populations. Math Biosci. 29, 85 -98 (1976)

8. Miller, R. S.: Pattern and process in competition. Advances in Ecological Research 4, 1 - 74 (1976)

9. Solobodkin, L. B.: Growth and regulation of animal populations, p. 167. Winston, N.Y.: HoltRinehart 1961

10. Verhulst, P. F.: Notice sur la loi que la population pursuit dans son accroissement. Correspond. Math. Phys. 10, 113-121 (1838)

11. Volterra, V.: Variations and fluctuations of the number of individuals of animal species living together. Animal Ecology (R. N. Chapman, ed.). New York: McGraw-Hill 1926

12. Windsor, C. P.: Mathematical analysis of the growth of mixed populations. Cold Spring Harb. Sym. Quant. Biol. 2, 181 - 187 (1934)

Received May 25/Revised October 12, 1980 